\title{
Inventory model with different demand rate and different holding cost
}

\author{
R.P. Tripathi*
}

Graphic Era University, Dehradun (UK), India

\section{CHRON I C LE}

Article history:

Received January 22013

Received in revised format

March 102013

Accepted March 102013

Available online

March 102013

Keywords:

Inventory model

Time-varying demand rate

Time-dependent holding cost

Optimization

\section{A B S T R A C T}

\begin{abstract}
This paper deals with the development of an inventory model for time varying demand and constant demand; and time dependent holding cost and constant holding cost for case 1 and case 2 respectively. Previous models incorporating that the holding cost is constant for the entire inventory cycle. Mathematical model has been developed for determining the optimal order quantity, the optimal cycle time and optimal total inventory cost for both cases. Differential calculus is used for finding optimal solution. Numerical examples are given for both cases to validate the proposed model. Sensitivity analysis is carried out to analyze the effect of changes in the optimal solution with respect to change in various parameters.
\end{abstract}

(C) 2013 Growing Science Ltd. All rights reserved

\section{Introduction}

It is important to find the optimal stock and optimal cycle time of inventory to meet the future demand. The main objective of inventory management is to minimize the inventory carrying cost. In traditional EOQ model, the demand rate is assumed to be constant. In real life, it is frequently observed that demand for a particular product can be influenced by internal factors such as price, time and availability. The change in the demand in response to inventory or marketing decisions is as demand elasticity. Thus, when the demand rate is constant, the effect of variability of the holding cost of the total inventory cost functions of such models has also been considered. Two types of demand and holding cost have been considered (i) time-dependent demand rate and time-dependent holding cost for case 1 (ii) constant demand rate and constant holding cost for case 2. An algorithm that minimizes the total inventory cost is developed.

Various models have been proposed for constant demand rate with constant holding cost. Teng et al. (2005) developed an EOQ model on optimal pricing and ordering policy under permissible delay in payments by assuming that the selling price is necessarily higher than the purchase cost. They established an appropriate model for a retailer to found its optimal price and lot size, simultaneously,

* Corresponding author.

E-mail: tripathi_rp0231@rediffmail.com (R.P.Tripathi)

(C) 2013 Growing Science Ltd. All rights reserved.

doi: $10.5267 /$ j.ijiec.2013.03.001 
when the supplier offered a permissible delay in payment. Goyal (1985) developed an EOQ model under permissible delay in payments but ignored the difference between the selling price and the purchase cost. Aggarwal and Jaggi (1995) extended Goyal's model for deteriorating items and Liao et al. (2000) developed an EOQ model for stock-dependent demand rate under permissible delay in payment. Muhlemann and Valtis-Spanopoulos (1980) investigated the constant rate EOQ model but with variable holding cost expressed as a percentage of the average value of capital investigated in stock. Vander Veen (1967) presented an EOQ inventory system with the holding cost as a nonlinear function of inventory. Weiss (1982) investigated traditional EOQ model with the holding cost per unit modified as a nonlinear function of the length of time an item was held in stock. Goh (1994) presented an EOQ model with general demand and holding cost function and demand rate for an item was considered as a function of existing inventory level and carrying cost per unit was allowed to change.

Alfares (2007) presented the step structure of the holding cost by considering the inventory policy for an item with a stock-level dependent demand rate and a storage-time dependent holding cost. The holding cost per unit of item per unit time was assumed an increasing function of the time spent in storage. The holding cost was assumed to be varying over time in only few inventory models. Giri et al. (1996) presented generalized EOQ model for deteriorating items with shortages, in which both the demand rate and the holding cost were continuous function of time. Datta and Pal (1990) developed an infinite time horizon deterministic inventory system without shortage, which has a level-dependent demand rate up to a certain stock-level and a constant demand for the rest of the cycle. Pal et al. (1993) presented a deterministic inventory model assuming that the demand rate was stock-dependent and that the items deteriorate at a constant rate. The total profit over one production run is maximized by numerically solving two non - linear equations.

In the study of EOQ model, the effect of inflation cannot be ignored. In this direction Hou and Lin (2009) presented a cash flow oriented EOQ model with deteriorating items under permissible delay in payments. In this study, they also considered cash flow as part of their modeling formulation. Tripathi et al. (2010) extended Hou and Lin (2009) model by considering time-dependent demand rate. Liao et al. (2000) presented an inventory model with deteriorating items under inflation and permissible delay in payment presented. Liao et al. (2000) developed an inventory model for initial stock-dependent consumption rate when a delay in payment was permissible. Hou et al. (2006) developed an inventory model for deteriorating items with stock-dependent consumption rate and shortages under inflation and time discounting. Hou (2006) presented a finite planning horizon inventory model for deterioration items with stock-dependent consumption rate and shortages with the effect of inflation and time-value of money on replenishment policy. In this direction, Jaggi et al. (2007) presented a model retailer's optimal ordering policy under two-stage trade credit financing. They also developed an inventory model under two levels of trade credit policy by assuming the demand was a function of credit period offered by the retailer to the customers using discounted cash flow (DCF) approach. A DCF approach permits a proper recognition of the timing of cash flows connected with an inventory system under the trade credit. Dye et al. (2007) investigated inventory and pricing strategies for deteriorating items with shortages using a discounted cash flow approach. They found the optimal inventory and pricing strategies maximizing the net present value of the total profit over the infinite horizon. Chung and Liao (2009) developed an optimal ordering policy of EOQ model under trade credit depending on the ordering quantity from the DCF approach. They discussed the optimal order quantity of the EOQ model that is not only dependent on the inventory policy but also on firm credit policy using discounted cash- flow (DCF) approach and trade credit depending on the quantity ordered.

Researchers in the past have established their inventory lot-size models under trade credit financing by assuming that the demand rate is constant (Jaggi et al., 2011; Hsu, 2012; Roy et al., 2012). Recently, Teng et al. (2012) established an EOQ model with trade credit financing for non-decreasing demand and optimal solution and relevant managerial phenomena was also calculated. An EOQ model with delay in payments and time varying deterioration rate was discussed and developed by Sarkar (2012) where the retailers were allowed a trade-credit offer by suppliers to buy more items with different 
discount rates on the purchasing cost. Hung (2011) developed an inventory model with generalized type demand, deterioration and backorder rates. Hung (2011) extended their model from ramp type demand rate and Weibull deterioration rate to arbitrary demand rate and arbitrary deterioration rate in the consideration of partial backorder. Khanra et al. (2011) presented an EOQ model for a deteriorating item with time-dependent quadratic demand under permissible delay in payment. In this paper, an effort has been made to analyze an EOQ model for deteriorating item considering quadratic time dependent demand rate and permissible delay in payment. Sana (2010) formulated optimal selling price and lot size with time varying deterioration and partial backlogging. In this work, an EOQ model over an infinite time horizon for perishable item where demand is price dependent and partial backorder permitted is discussed. Deterministic inventory model for deteriorating items with trade credit financing and capacity constraints is developed by Liao and Huang (2010) and they presented an inventory model for optimizing the replenishment cycle time for a single deteriorating item under a permissible delay in payments and constraints on warehouse capacity.

In this paper, we consider the demand rate is time varying and holding cost is time-dependent for case 1 ; and demand rate and holding cost both are constant for case 2. The main objective of this paper is to obtain minimum total inventory cost (TIC), order quantity and corresponding order cycle for both cases. The remainder of the paper is organized as follows. Relevant notation and assumptions are given in the next section. This is followed by mathematical formulation in the section 3. Algorithm and numerical example is given in section 4 and 5, respectively followed by sensitivity analysis is in section 6 . Finally, suggestions and concluding remarks are given in section 7.

\section{Notations and Assumptions}

The following notations are used throughout the manuscript:

$\begin{array}{ll}\mathrm{k} & : \text { ordering cost per order } \\ \lambda_{0} & : \text { constant annual demand rate } \\ \mathrm{I}(\mathrm{t}) & : \text { on-hand inventory level at time ' } \mathrm{t} \text { ' } \\ \mathrm{h} & : \text { holding cost of the item for case } 2 \\ \mathrm{~h}(\mathrm{t}) & : \text { time dependent holding cost of the item at time } \mathrm{t}, \mathrm{h}(\mathrm{t}) \equiv \mathrm{h} . \mathrm{t} \\ \mathrm{T} & : \text { cycle time } \\ \beta & : \text { demand parameter indicating elasticity, } 0<\beta<1 \\ \mathrm{R}(\mathrm{t}) & : \text { time varying demand i.e. } \mathrm{R}(\mathrm{t})=\lambda_{0} \mathrm{t}^{-\beta}, \text { for case } 1 \quad \lambda_{0}>0, \quad 0<\beta<1, \quad 0 \leq \mathrm{t} \leq \mathrm{T} \\ \mathrm{T}^{*} & : \text { optimal cycle time foe case } 1 \\ \mathrm{~T}^{* *} & : \text { optimal cycle time foe case } 2 \\ \mathrm{TIC} & : \text { total inventory cost per cycle } \\ \mathrm{TIC}^{*} & : \text { optimal total inventory cost per cycle for case } 1 \\ \mathrm{TIC}^{* *} & : \text { optimal total inventory cost per cycle for case } 2 \\ \mathrm{TIC}_{1} * & : \text { optimal total inventory cost per cycle for case } 1 \text { as } \beta \rightarrow 0 \\ \mathrm{Q}^{*} & : \text { ordering quantity } \\ \mathrm{Q}_{1}{ }^{*} & : \text { optimal ordering quantity for case } 1 \\ \mathrm{Q}_{2}{ }^{*} & : \text { optimal ordering quantity for case } 2 \\ \mathrm{Q}_{11}{ }^{*} & : \text { optimal ordering quantity as } \beta \rightarrow 0\end{array}$

In addition, the following assumptions are being made to develop aforesaid model:

1. The demand rate $\mathrm{R}(\mathrm{t})$ is decreasing function of time with increase of ' $\beta$ ' for case 1

2. The demand $\lambda_{0}$ rate is constant for case 2

3. The holding cost is time dependent and holding cost parameter ' $h$ ' i.e. $h(t) \equiv h . t$

4. Shortages are not allowed

5. The inventory system under consideration deals with single item 
6. The planning horizon in infinite and lead time is zero

7. The demand rate (for case 1$) \mathrm{R}(\mathrm{t})$ is decreasing function of time is expressed as $\mathrm{R}(\mathrm{t})=\lambda_{0} t^{-\beta}, \lambda_{0}>0, \quad 0<\beta<1, \quad 0 \leq \mathrm{t} \leq \mathrm{T}$

\section{Mathematical Formulation}

The objective is to minimize the total inventory cost (TIC) per unit time, which contains two components: (a) the ordering cost and (b) the holding cost. The ordering cost per unit time is (k/T), since one order is made per cycle. The total holding cost per cycle is the integral of the product of the holding cost $\mathrm{h}(\mathrm{t})$ and inventory level $\mathrm{I}(\mathrm{t})$ over the whole cycle ' $\mathrm{T}$ '.

$$
\text { TIC }=\frac{k}{T}+\frac{1}{T} \int_{0}^{T} h(t) \cdot I(t) d t
$$

Since the demand rate is equal to the rate of inventory level decrease, the rate of change of inventory level is governed by the following differential equation:

$$
\frac{d I(t)}{d t}=-\lambda_{0} t^{-\beta}, \lambda_{0}>0, \quad 0<\beta<1, \quad 0 \leq \mathrm{t} \leq \mathrm{T}
$$

The on-hand inventory level at time ' $t$ ' $I(t)$, can be evaluated on solving (3) with the initial condition $\mathrm{I}(\mathrm{T})=0$, we obtain

$$
I(t)=\frac{\lambda_{0}}{(1-\beta)}\left(\mathrm{T}^{1-\beta}-\mathrm{t}^{1-\beta}\right)
$$

and the order quantity is

$$
\mathrm{Q}=\frac{\lambda_{0} T^{1-\beta}}{1-\beta}, \quad 0<\beta<1
$$

From (3), we obtain

$$
T=\left\{\frac{(1-\beta) Q}{\lambda_{0}}\right\}^{1 /(1-\beta)}
$$

\subsection{Case 1. Time dependent demand rate and Time dependent holding cost}

In this case, holding cost $h$ is assumed to be an increasing step function of storage time. The holding cost depends on the length of the storage used in this case. The total inventory cost per unit time is expressed as

TIC $=\frac{k}{T}+\frac{1}{T} \int_{0}^{T} h \cdot t \cdot I(t) d t=\frac{k}{T}+\frac{h \lambda_{0} T^{2-\beta}}{2(3-\beta)}$

Using Eq. (6) in Eq. (7) yields,

$$
\begin{aligned}
& T I C=k Q^{-1 /(1-\beta)}\left(\frac{\lambda_{0}}{1-\beta}\right)^{1 /(1-\beta)}+\frac{h \lambda_{0}}{2(3-\beta)} Q^{(2-\beta) /(1-\beta)}\left(\frac{1-\beta}{\lambda_{0}}\right)^{(2-\beta) /(1-\beta)} \\
& \frac{d(T I C)}{d Q}=-\frac{k Q^{-(2-\beta) /(1-\beta)}}{(1-\beta)}\left(\frac{\lambda_{0}}{1-\beta}\right)^{1 /(1-\beta)}+\frac{h \lambda_{0}(2-\beta)}{2(3-\beta)(1-\beta)} Q^{1 /(1-\beta)}\left(\frac{1-\beta}{\lambda_{0}}\right)^{(2-\beta) /(1-\beta)}
\end{aligned}
$$

$\frac{d^{2}(\mathrm{TIC})}{d Q^{2}}=\left[\frac{k(2-\beta) Q^{-(3-2 \beta) /(1-\beta)}}{(1-\beta)^{2}}\left(\frac{\lambda_{0}}{1-\beta}\right)^{1 /(1-\beta)}+\frac{h \lambda_{0}(2-\beta)}{2(3-\beta)(1-\beta)^{2}} Q^{\beta /(1-\beta)}\left(\frac{1-\beta}{\lambda_{0}}\right)^{(2-\beta) /(1-\beta)}\right]>0$ 
The optimal (minimum) $\mathrm{Q}=\mathrm{Q}^{*}$ is obtained by solving $\frac{d(T I C)}{d Q}=0$, for $\mathrm{Q}$, we obtain

$Q=Q^{*}=\frac{\lambda_{0}^{2 /(3-\beta)}}{1-\beta}\left\{\frac{2 k(3-\beta)}{h(2-\beta)}\right\}^{(1-\beta) /(3-\beta)}$

If $\beta \rightarrow 0$, the optimal $\mathrm{Q}=\mathrm{Q}^{*}=\mathrm{Q}_{11}$ * reduces to

$Q=Q_{1}^{*}=\lambda_{0}^{2 / 3}\left(\frac{3 k}{h}\right)^{1 / 3}$

and $\mathrm{TIC}=\mathrm{TIC}_{1} *=\frac{k}{T}+\frac{h \lambda_{0} T^{2}}{6}$

\subsection{Case 2. Constant demand rate and constant holding cost}

The objective is to minimize the total inventory cost (TIC) per unit time. The total inventory cost (TIC) contains the same components as stated in case 1. Since the demand rate is equal to the rate of inventory level decrease, we can describe $\mathrm{I}(\mathrm{t})$ by the following differential equation:

$\frac{d I(t)}{d t}=-\lambda_{0} \quad, \lambda_{0}>0,0 \leq t \leq T$

The solution of (13) with $\mathrm{I}(\mathrm{T})=0$, is given by

$I(t)=\lambda_{0}(T-t), 0 \leq t \leq T$

The order quantity $\mathrm{Q}=\mathrm{I}(0)=\lambda_{0} T$

Thus, $T=\frac{Q}{\lambda_{0}}$.

In this case, the TIC per unit time can be expresses as

TIC $=\frac{k}{T}+\frac{1}{T} \int_{0}^{T} h \cdot I(t) d t$

Substituting I(t) from Eq. (15) into Eq. (18) yields,

TIC $=\frac{k}{T}+\frac{1}{T} \int_{0}^{T} h \cdot \lambda_{0}(T-t) d t=\frac{k}{T}+\frac{h \lambda_{0} T}{2}$

Substituting the values of T from Eq. (17) into Eq. (19) yields,

$\operatorname{TIC}(Q)=\frac{k \lambda_{0}}{Q}+\frac{h Q}{2}$

Differentiating (20) w.r.t. 'Q' two times, we obtain

$\frac{d T I C}{d Q}=-\frac{k \lambda_{0}}{Q^{2}}+\frac{h}{2}$ and $\frac{d^{2} T I C}{d Q^{2}}=\frac{2 k \lambda_{0}}{Q^{3}}>0$

The optimal (minimum) $\mathrm{Q}=\mathrm{Q}_{2} *$ is obtained by solving $\frac{d(T I C)}{d Q}=0$ from (21) for $\mathrm{Q}$, we obtain

$Q=Q_{2}^{*}=\sqrt{\frac{2 k \lambda_{0}}{h}}$

\section{Numerical Examples}

Example 1: Let $\lambda_{0}=500$ units/ year, $\mathrm{k}=\$ 400$ per order, $\beta=0.1$. 
Table 1

Values of optimal $\mathrm{T}=\mathrm{T} *, \mathrm{Q}=\mathrm{Q}_{1}{ }^{*}$ and $\mathrm{TIC}=\mathrm{TIC}^{*}$ for different values of ' $\mathrm{h}$ '

\begin{tabular}{cccccccc}
\hline $\mathrm{h}$ & $\mathrm{Q}=\mathrm{Q}^{*}$ & $\mathrm{~T}=\mathrm{T}^{*}$ & $\mathrm{TIC}=\mathrm{TIC}$ & $\mathrm{h}$ & $\mathrm{Q}=\mathrm{Q}_{1}^{*}$ & $\mathrm{~T}=\mathrm{T}^{*}$ & $\mathrm{TIC}=\mathrm{TIC}$ \\
\hline 10 & 358.693 & 0.615012 & 992.705 & 37 & 238.993 & 0.391699 & 1558.661 \\
11 & 348.239 & 0.595129 & 1025.873 & 38 & 237.023 & 0.388113 & 1573.061 \\
12 & 338.961 & 0.577538 & 1057.119 & 39 & 235.120 & 0.384653 & 1587.214 \\
13 & 330.645 & 0.561816 & 1086.703 & 40 & 233.280 & 0.381310 & 1601.132 \\
14 & 323.127 & 0.547640 & 1114.831 & 41 & 231.499 & 0.378076 & 1614.823 \\
15 & 316.282 & 0.534765 & 1141.672 & 42 & 229.774 & 0.374947 & 1628.297 \\
16 & 310.010 & 0.522996 & 1167.364 & 43 & 228.102 & 0.371917 & 1641.563 \\
17 & 304.232 & 0.512176 & 1192.025 & 44 & 226.481 & 0.368982 & 1654.628 \\
18 & 298.883 & 0.502180 & 1215.752 & 45 & 224.907 & 0.366133 & 1667.914 \\
19 & 293.909 & 0.492903 & 1238.631 & 46 & 223.378 & 0.363369 & 1680.186 \\
20 & 289.268 & 0.484263 & 1260.734 & 47 & 221.892 & 0.360684 & 1692.692 \\
21 & 284.921 & 0.476184 & 1282.124 & 48 & 220.447 & 0.358075 & 1705.026 \\
22 & 280.837 & 0.468606 & 1302.857 & 49 & 219.041 & 0.355538 & 1717.192 \\
23 & 276.989 & 0.461477 & 1322.982 & 50 & 217.672 & 0.353070 & 1729.196 \\
24 & 273.355 & 0.454755 & 1342.541 & 51 & 216.338 & 0.350670 & 1741.044 \\
25 & 269.914 & 0.448399 & 1361.573 & 52 & 215.038 & 0.348326 & 1752.741 \\
26 & 266.648 & 0.442374 & 1380.112 & 53 & 213.771 & 0.346047 & 1764.292 \\
27 & 263.543 & 0.436654 & 1398.190 & 54 & 212.534 & 0.343823 & 1775.700 \\
28 & 260.585 & 0.431212 & 1415.835 & 55 & 211.327 & 0.341654 & 1786.971 \\
29 & 257.763 & 0.426027 & 1433.071 & 56 & 210.149 & 0.339538 & 1798.109 \\
30 & 255.065 & 0.421075 & 1449.922 & 57 & 208.997 & 0.337471 & 1809.117 \\
31 & 252.483 & 0.416341 & 1466.409 & 58 & 207.873 & 0.335455 & 1820.000 \\
32 & 250.007 & 0.411807 & 1482.551 & 59 & 206.773 & 0.333483 & 1830.759 \\
33 & 247.631 & 0.407461 & 1498.366 & 60 & 205.697 & 0.331555 & 1841.400 \\
34 & 245.347 & 0.403287 & 1513.870 & 65 & 200.650 & 0.322529 & 1892.934 \\
35 & 243.150 & 0.399277 & 1529.078 & 70 & 196.088 & 0.314392 & 1941.928 \\
36 & 241.034 & 0.395418 & 1544.004 & 80 & 188.128 & 0.300244 & 2033.434 \\
\hline
\end{tabular}

Table 2

\begin{tabular}{|c|c|c|c|c|c|c|c|}
\hline $\mathrm{h}$ & $\mathrm{Q}=\mathrm{Q}_{2} *$ & $\mathrm{~T}=\mathrm{T}^{* *}$ & $\mathrm{TIC}=\mathrm{TIC}^{* *}$ & $\mathrm{~h}$ & $\mathrm{Q}=\mathrm{Q}_{2} *$ & $\mathrm{~T}=\mathrm{T}^{* *}$ & $\mathrm{TIC}=\mathrm{TIC}^{* *}$ \\
\hline 1 & 632.456 & 1.264911 & 632.456 & 36 & 105.409 & 0.210818 & 3794.733 \\
\hline 5 & 282.843 & 0.565685 & 1414.214 & 37 & 103.975 & 0.207950 & 3847.0768 \\
\hline 10 & 200.000 & 0.400000 & 2000.000 & 38 & 102.598 & 0.205200 & 3898.718 \\
\hline 11 & 190.693 & 0.381385 & 2097.618 & 39 & 101.274 & 0.202548 & 3949.684 \\
\hline 12 & 182.574 & 0.365148 & 2190.890 & 40 & 100.000 & 0.200000 & 4000.000 \\
\hline 13 & 175.412 & 0.350823 & 2280.351 & 41 & 98.773 & 0.197546 & 4049.691 \\
\hline 14 & 169.031 & 0.338062 & 2366.432 & 42 & 97.590 & 0.195180 & 4098.780 \\
\hline 15 & 163.299 & 0.326599 & 2449.490 & 43 & 96.449 & 0.192897 & 4147.288 \\
\hline 16 & 158.114 & 0.316228 & 2529.822 & 44 & 95.346 & 0.190693 & 4195.235 \\
\hline 17 & 153.393 & 0.306786 & 2607.681 & 45 & 94.281 & 0.188562 & 4242.641 \\
\hline 18 & 149.071 & 0.298142 & 2683.282 & 46 & 93.250 & 0.186501 & 4289.522 \\
\hline 19 & 145.095 & 0.290191 & 2756.810 & 47 & 92.253 & 0.184506 & 4335.897 \\
\hline 20 & 141.421 & 0.282843 & 2828.427 & 48 & 91.287 & 0.182574 & 4381.780 \\
\hline 21 & 138.013 & 0.276026 & 2898.275 & 49 & 90.351 & 0.180702 & 4427.189 \\
\hline 22 & 134.840 & 0.269680 & 2966.479 & 50 & 89.443 & 0.178885 & 4472.136 \\
\hline 23 & 131.876 & 0.263752 & 3033.150 & 51 & 88.561 & 0.177123 & 4516.636 \\
\hline 24 & 129.099 & 0.258199 & 3098.387 & 52 & 87.706 & 0.175412 & 4560.702 \\
\hline 25 & 126.491 & 0.252982 & 3162.278 & 53 & 86.874 & 0.173749 & 4604.346 \\
\hline 26 & 124.035 & 0.248069 & 3224.903 & 54 & 86.0663 & 0.172133 & 4647.580 \\
\hline 27 & 121.716 & 0.243432 & 3286.335 & 55 & 85.280 & 0.170561 & 4690.416 \\
\hline 28 & 119.523 & 0.239046 & 3346.640 & 56 & 84.515 & 0.169031 & 4732.864 \\
\hline 29 & 117.444 & 0.234888 & 3405.877 & 57 & 83.771 & 0.167542 & 4774.934 \\
\hline 30 & 115.470 & 0.230940 & 3464.102 & 58 & 83.045 & 0.166091 & 4816.638 \\
\hline 31 & 113.592 & 0.227185 & 3521.363 & 59 & 82.339 & 0.164677 & 4847.983 \\
\hline 32 & 111.803 & 0.223607 & 3577.709 & 60 & 81.650 & 0.163299 & 4898.979 \\
\hline 33 & 110.096 & 0.220193 & 3633.180 & 65 & 78.446 & 0.156893 & 5999.018 \\
\hline 34 & 108.465 & 0.216930 & 3687.818 & 70 & 75.593 & 0.151186 & 5291.499 \\
\hline 35 & 106.904 & 0.213809 & 3741.657 & 80 & 70.711 & 0.141421 & 5656.861 \\
\hline
\end{tabular}

\section{Sensitivity Analysis}

To find sensitivity analysis, the effect of parameters ' $h$ ', ' $k$ ' and ' $\beta$ ' on the optimal solution, the set of values of ' $h$ ', ' $k$ ' and ' $\beta$ ' are assumed to as $\mathrm{h}=60,55,50,45,40,35$, ' $\mathrm{k}$ ' $=410,420,430,440,450$, 460,470 , and 480 , and ' $\beta$ ' $=0.2,0.3,0.4,0.5,0.6,0.7,0.8$, and 0.9 . 
Meanwhile the other parameter values follows those data mentioned above in the numerical example 1. The results of sensitivity analysis are given in Tables 3,4 , and 5 .

\section{(i) Sensitivity Analysis: (for case 1).}

\section{Table 3}

Variation of optimal solution of $\mathrm{Q}=\mathrm{Q}_{1}{ }^{*}, \mathrm{~T}=\mathrm{T}^{*}$ and TIC $=\mathrm{TIC} *$ with the variation of ' $\mathrm{h}$ ' and ' $\beta$ ', keeping all the parameters same as in Example 1.

\begin{tabular}{|c|c|c|c|c|c|c|c|c|c|}
\hline $\mathrm{h}$ & $\beta \rightarrow$ & 0.2 & 0.3 & 0.4 & 0.5 & 0.6 & 0.7 & 0.8 & 0.9 \\
\hline \multirow{3}{*}{60} & Q & 251.757 & 314.687 & 403.868 & 536.492 & 747.455 & 1119.077 & 1900.104 & 4338.992 \\
\hline & $\mathrm{T}$ & 0.320905 & 0.310055 & 0.299021 & 0.287824 & 0.276495 & 0.265076 & 0.253622 & 0.242208 \\
\hline & TIC & 1938.962 & 2048.974 & 2173.765 & 2316.230 & 2480.018 & 2669.768 & 2891.445 & 3155.787 \\
\hline \multirow{3}{*}{55} & $\mathrm{Q}$ & 258.094 & 321.866 & 412.059 & 545.910 & 758.374 & 1131.850 & 1915.193 & 4357.007 \\
\hline & $\mathrm{T}$ & 0.331034 & 0.320209 & 0.309196 & 0.298018 & 0.286704 & 0.275296 & 0.263853 & 0.252454 \\
\hline & TIC & 1879.633 & 1983.996 & 2102.222 & 2237.002 & 2391.716 & 2570.654 & 2779.319 & 3024.850 \\
\hline \multirow{3}{*}{50} & Q & 265.219 & 329.919 & 421.223 & 556.416 & 770.517 & 1146.009 & 1931.86 & 4376.827 \\
\hline & $\mathrm{T}$ & 0.342492 & 0.331715 & 0.320742 & 0.309599 & 0.298319 & 0.286944 & 0.275536 & 0.264176 \\
\hline & TIC & 1816.728 & 1915.182 & 2091.508 & 2153.323 & 2298.596 & 2466.306 & 2661.482 & 2890.633 \\
\hline \multirow{3}{*}{45} & $\mathrm{Q}$ & 273.324 & 339.055 & 431.590 & 568.265 & 784.167 & 1161.867 & 1950.452 & 4398.841 \\
\hline & $\mathrm{T}$ & 0.355629 & 0.344915 & 0.334006 & 0.322925 & 0.311707 & 0.300395 & 0.289052 & 0.277768 \\
\hline & TIC & 1749.637 & 1841.886 & 1946.073 & 2064.459 & 2199.870 & 2305.882 & 2537.0244 & 2749.103 \\
\hline \multirow{3}{*}{40} & Q & 282.679 & 349.568 & 443.482 & 581.811 & 799.712 & 1179.854 & 1971.449 & 4423.582 \\
\hline & $\mathrm{T}$ & 0.370909 & 0.360294 & 0.349485 & 0.338504 & 0.327385 & 0.316178 & 0.304949 & 0.293793 \\
\hline & TIC & 1677.565 & 1763.565 & 1859.881 & 1960.045 & 2094.515 & 2238.268 & 2452.032 & 2599.234 \\
\hline \multirow{3}{*}{35} & Q & 293.672 & 361.882 & 457.360 & 597.558 & 817.710 & 1200.584 & 1995.527 & 4451.800 \\
\hline & $\mathrm{T}$ & 0.389026 & 0.378551 & 0.367902 & 0.357076 & 0.346117 & 0.335078 & 0.324032 & 0.313081 \\
\hline & TIC & 1599.440 & 1678.182 & 1766.772 & 1867.018 & 1981.163 & 2112.021 & 2263.151 & 2439.103 \\
\hline \multirow{3}{*}{30} & Q & 306.895 & 376.638 & 473.923 & 616.268 & 838.990 & 1224.968 & 2023.688 & 4484.599 \\
\hline & $\mathrm{T}$ & 0.411043 & 0.400804 & 0.390375 & 0.379786 & 0.369077 & 0.358305 & 0.347550 & 0.336927 \\
\hline & TIC & 1513.764 & 1583.152 & 1665.067 & 1755.374 & 1857.914 & 1975.108 & 2110.003 & 2266.474 \\
\hline
\end{tabular}

\section{(ii) Sensitivity Analysis: (for case 1)}

\section{Table 4}

Variation of optimal solution of $\mathrm{Q}=\mathrm{Q}^{*}, \mathrm{~T}^{*}$ and $\mathrm{TIC}^{*}$ with the variation of ' $\mathrm{h}$ ' and ' $\mathrm{k}$ ', keeping all the parameters same as in Example 1.

\begin{tabular}{cccccccccc}
\hline $\begin{array}{l}\mathrm{h} \\
\downarrow\end{array}$ & $\mathrm{k} \rightarrow$ & 410 & 420 & 430 & 440 & 450 & 460 & 470 & 480 \\
\hline \multirow{2}{*}{60} & $\mathrm{Q}$ & 207.279 & 208.835 & 210.366 & 211.872 & 213.355 & 214.815 & 216.254 & 217.672 \\
& $\mathrm{~T}$ & 0.33439 & 0.337181 & 0.339928 & 0.342633 & 0.345299 & 0.347926 & 0.350516 & 0.353069 \\
& $\mathrm{TIC}$ & 1871.435 & 1901.212 & 1930.750 & 1960.053 & 1989.123 & 2017.974 & 2046.608 & 2075.037 \\
\hline \multirow{2}{*}{55} & $\mathrm{Q}$ & 212.953 & 214.552 & 216.124 & 217.672 & 219.195 & 220.695 & 222.173 & 223.630 \\
& $\mathrm{~T}$ & 0.344576 & 0.347451 & 0.350282 & 0.353069 & 0.355816 & 0.358523 & 0.361192 & 0.363823 \\
& $\mathrm{TIC}$ & 1787.094 & 1845.016 & 1873.679 & 1902.117 & 1930.329 & 1958.327 & 1986.114 & 2013.702 \\
\hline \multirow{2}{*}{50} & $\mathrm{Q}$ & 219.346 & 220.993 & 222.612 & 224.206 & 225.775 & 227.321 & 228.843 & 230.343 \\
& $\mathrm{~T}$ & 0.356089 & 0.359060 & 0.361985 & 0.364866 & 0.367704 & 0.370502 & 0.373260 & 0.375979 \\
& $\mathrm{TIC}$ & 1757.397 & 1785.364 & 1813.102 & 1840.618 & 1867.920 & 1895.011 & 1921.901 & 1948.596 \\
\hline \multirow{2}{*}{45} & $\mathrm{Q}$ & 226.637 & 228.338 & 230.012 & 231.658 & 233.280 & 234.876 & 236.449 & 237.999 \\
& $\mathrm{~T}$ & 0.369264 & 0.372345 & 0.375378 & 0.378366 & 0.381309 & 0.384210 & 0.387070 & 0.389890 \\
& $\mathrm{TIC}$ & 1694.695 & 1721.663 & 1748.413 & 1774.946 & 1801.274 & 1827.399 & 1853.330 & 1879.072 \\
\hline \multirow{2}{*}{40} & $\mathrm{Q}$ & 235.074 & 236.838 & 238.575 & 240.283 & 241.965 & 243.621 & 245.252 & 246.860 \\
& $\mathrm{~T}$ & 0.38457 & 0.387779 & 0.390938 & 0.394049 & 0.397115 & 0.400136 & 0.403114 & 0.406052 \\
& $\mathrm{TIC}$ & 1627.245 & 1653.139 & 1678.823 & 1704.302 & 1729.580 & 1754.666 & 1779.566 & 1804.281 \\
\hline \multirow{2}{*}{35} & $\mathrm{Q}$ & 245.021 & 246.860 & 248.669 & 250.450 & 252.203 & 253.929 & 255.629 & 257.305 \\
& $\mathrm{~T}$ & 0.402692 & 0.406052 & 0.412618 & 0.412618 & 0.415828 & 0.418991 & 0.422110 & 0.425186 \\
& $\mathrm{TIC}$ & 1554.016 & 1578.746 & 1627.605 & 1627.605 & 1651.746 & 1675.705 & 1699.482 & 1723.086 \\
\hline \multirow{2}{*}{30} & $\mathrm{Q}$ & 257.027 & 258.957 & 260.855 & 262.722 & 264.561 & 266.372 & 268.156 & 269.914 \\
& $\mathrm{~T}$ & 0.424676 & 0.428219 & 0.431708 & 0.435144 & 0.438529 & 0.441865 & 0.445154 & 0.448398 \\
& $\mathrm{TIC}$ & 1473.569 & 1497.020 & 1520.277 & 1543.349 & 1566.241 & 1588.958 & 1611.506 & 1633.887 \\
\hline
\end{tabular}


(iii)Sensitivity Analysis: (for case 2).

\section{Table 5}

Variation of optimal solution of $\mathrm{Q}=\mathrm{Q}_{2}{ }^{*}, \mathrm{~T}=\mathrm{T} * *$ and $\mathrm{TIC}=\mathrm{TIC} * *$ with the variation of ' $\mathrm{h}$ ' and ' $\mathrm{k}$ ', keeping all the parameters same as in Example 1.

\begin{tabular}{cccccccccc}
\hline $\mathrm{h}$ & $\mathrm{k} \rightarrow$ & 410 & 420 & 430 & 440 & 450 & 460 & 470 & 480 \\
$\downarrow$ & & & & & & & & \\
\hline \multirow{2}{*}{60} & $\mathrm{Q}$ & 82.664 & 83.666 & 84.656 & 85.635 & 86.603 & 87.560 & 88.506 \\
& $\mathrm{~T}$ & 0.165328 & 0.167332 & 0.169312 & 0.171270 & 0.173205 & 0.175119 & 0.177012 & 0.178885 \\
& $\mathrm{TIC}$ & 4959.838 & 5019.960 & 5079.375 & 5138.090 & 5196.154 & 5253.570 & 5310.371 & 5366.570 \\
\hline \multirow{2}{*}{55} & $\mathrm{Q}$ & 86.340 & 87.386 & 88.421 & 89.443 & 90.453 & 91.453 & 92.442 & 93.420 \\
& $\mathrm{~T}$ & 0.172679 & 0.174773 & 0.176841 & 0.178885 & 0.180907 & 0.182906 & 0.184883 & 0.186840 \\
& $\mathrm{TIC}$ & 4748.690 & 4806.240 & 4863.126 & 4919.356 & 4974.934 & 5029.908 & 5084.293 & 5138.090 \\
\hline \multirow{2}{*}{50} & $\mathrm{Q}$ & 90.554 & 91.652 & 92.736 & 93.808 & 94.868 & 95.917 & 96.954 & 97.980 \\
& $\mathrm{~T}$ & 0.181108 & 0.183303 & 0.185472 & 0.187617 & 0.189737 & 0.191833 & 0.193907 & 0.195959 \\
& $\mathrm{TIC}$ & 4527.689 & 4582.576 & 4636.814 & 4690.411 & 4743.412 & 4795.835 & 4847.682 & 4898.982 \\
\hline \multirow{2}{*}{45} & $\mathrm{Q}$ & 95.452 & 96.609 & 97.753 & 98.883 & 100.000 & 101.105 & 102.198 & 103.280 \\
& $\mathrm{~T}$ & 0.190904 & 0.193218 & 0.195505 & 0.197765 & 0.200000 & 0.202210 & 0.204396 & 0.206559 \\
& $\mathrm{TIC}$ & 4295.349 & 4347.417 & 4398.864 & 4449.722 & 4500.000 & 4549.725 & 4598.914 & 4647.580 \\
\hline \multirow{2}{*}{40} & $\mathrm{Q}$ & 101.242 & 102.470 & 103.682 & 104.881 & 106.066 & 107.238 & 108.397 & 109.544 \\
& $\mathrm{~T}$ & 0.202484 & 0.204939 & 0.207364 & 0.209762 & 0.212132 & 0.214476 & 0.216795 & 0.219089 \\
& $\mathrm{TIC}$ & 4049.697 & 4098.780 & 4147.292 & 4195.233 & 4242.641 & 4289.523 & 4335.895 & 4381.781 \\
\hline \multirow{2}{*}{35} & $\mathrm{Q}$ & 108.233 & 109.544 & 110.841 & 112.122 & 113.389 & 114.642 & 115.882 & 117.108 \\
& $\mathrm{~T}$ & 0.216465 & 0.219089 & 0.221682 & 0.224245 & 0.226779 & 0.229285 & 0.231763 & 0.234216 \\
& $\mathrm{TIC}$ & 3788.140 & 3834.058 & 3879.432 & 3924.281 & 3968.624 & 4012.481 & 4055.864 & 4098.780 \\
\hline \multirow{2}{*}{30} & $\mathrm{Q}$ & 116.904 & 118.322 & 119.722 & 121.106 & 122.474 & 123.828 & 125.166 & 126.491 \\
& $\mathrm{~T}$ & 0.233809 & 0.236643 & 0.239444 & 0.242212 & 0.244949 & 0.247656 & 0.250333 & 0.252982 \\
& $\mathrm{TIC}$ & 3507.136 & 3549.649 & 3591.655 & 3633.181 & 3674.234 & 3714.833 & 3754.998 & 3794.735 \\
\hline
\end{tabular}

Based on the results we can make the following conclusions,

(a) Based on the observations found from Table 1 we can conclude that the optimal total inventory cost TIC* is directly associated with holding cost whereas the optimal order quantity $\mathrm{Q}^{*}$ and optimal cycle time $\mathrm{T}^{*}$ are inversely associated with holding cost.

(b) Based on the results of Table 2 we can conclude that the optimal total inventory cost TIC** is directly associated with holding cost ' $h$ ' whereas the optimal order quantity $\mathrm{Q}_{2}{ }^{*}$ and optimal cycle time $\mathrm{T}^{* *}$ is inversely associated with holding cost ' $h$ '.

(c) The observation found from the Table 3 are as follows,

(i) Any increase on ' $\beta$ ' results to an increase in optimal order quantity $Q^{*}$, TIC*, whereas any decrease on optimal cycle time $T^{*}$ does not change holding cost ' $h$ '.

(ii) Any increase on holding cost ' $h$ ' increases the optimal order quantity $Q^{*}$, optimal cycle time $\mathrm{T}^{*}$, whereas it decreases the total inventory cost TIC*.

(d) From Table 4, it can be easily seen that:

(i) An increase on ordering cost ' $\mathrm{k}$ ' results to an increase on optimal order quantity $\mathrm{Q}^{*}$, optimal cycle time $\mathrm{T}^{*}$ and optimal total inventory cost TIC', keeping holding cost ' $\mathrm{h}$ ' constant.

(ii) An increase on holding cost ' $h$ ' results to an increase on optimal order quantity $Q^{*}$, optimal cycle time $\mathrm{T}^{*}$, whereas it decreases the optimal total inventory cost TIC*.

(e) From Table 5, we observe that:

(i) An increase on ordering cost ' $\mathrm{k}$ ' results to an increase on optimal order quantity $\mathrm{Q}_{2}{ }^{*}$, optimal cycle time $\mathrm{T}^{* *}$, optimal total inventory cost $\mathrm{TIC}^{* *}$, keeping holding cost ' $h$ ' constant. 
(ii) An increase on holding cost ' $h$ ' results to an increase of optimal order quantity $\mathrm{Q}_{2}{ }^{*}$, optimal cycle time $\mathrm{T}^{* *}$, whereas it decreases the optimal total inventory cost $\mathrm{TIC}^{* *}$, keeping ordering cost ' $\mathrm{k}$ ' constant.

\section{Conclusion and Future Research}

In this paper, we have presented a new method for inventory system with time dependent demand and time dependent holding cost by considering two cases. The first case considers constant demand and holding cost is considered constant for the second case. Simple common optimization algorithm has been developed to find optimal solution and the proposed model has been examined using some numerical examples. The preliminary results indicate that the total inventory cost increases when we increase the holding cost ' $h$ '. It has also observed from the sensitivity analysis that the total inventory cost increases with the increase of ordering cost ' $k$ ' and ' $\beta$ ', whereas total inventory cost decreases with the increase of holding cost ' $h$ '

The model presented in this study can be extended in different ways. For instance, the model can be extended for variable ordering costs and non-instantaneous receipt of orders. This model can also extend for deteriorating items as well as shortages, freight charges etc.

Acknowledgments

The author would like to express his heartfelt thanks to anonymous referees and editor for their most valuable comments and constructive suggestions that have led to a significant improvement on an earlier version of this manuscript.

\section{References}

Aggarwal, S.P., \& Jaggi, C.K. (1995). Ordering policies of deteriorating items under permissible delay in payments. Journal of Operational Research Society, 46, 658-662.

Alfares, H.K. (2007). Inventory model with stock - level dependent demand rate and variable holding cost. International Journal of Production Economics, 108, 259 -265.

Chung, K.J., \& Liao, J.J. (2009). The optimal ordering policy of the EOQ model under trade credit depending on the ordering quantity from the DCF approach. European Journal of Operational Research, 196, $563-568$.

Datta, T.K., \& Pal, A.K. (1990). A note on inventory model with inventory level demand rate. Journal of the Operations Research Society, 41(10), 971-975.

Dye, C.Y., Ouyang, L.Y., \& Hsieh, T.P. (2007). Inventory and pricing strategies for deteriorating items with shortages: A discounted cash flow approach. Computers \& Industrial Engineering, 52, 29- 40.

Giri, B.C., Goswami, A., \& Chaudhuri, K.S. (1996). An EOQ model for deteriorating items with timevarying demand and costs. Journal of the Operational Research Society, 47(11), 1398- 1405.

Goh, M. (1994). EOQ model with general demand and holding cost function. European Journal of Operational Research, 73, 50-54.

Goyal, S.K (1985). Economic order quantity under conditions of permissible delay in payments. Journal of Operational Research Society, 36, 335-338.

Hou, K.L. (2006). An inventory model for deteriorating items with stock dependent consumption rate and shortages under inflation and time discounting. European Journal of operational Research, 168, 463- 474.

Hou, K.L., \& Lin, L.C. (2009). A cash flow oriented EOQ model with deteriorating items under permissible delay in payments. Journal of Applied Sciences, 9(9), 1791-1994.

Hung, K.C. (2011).An inventory model with generalized type demand, deterioration and backorder rates. European Journal of Operational Research, 208, 239- 242.

Hsu, L. F. (2012). A note on "An economic order quantity (EOQ) for items with imperfect quality and inspection errors". International Journal of Industrial Engineering Computations, 3(4), 695-702. 
Khanra, S. Ghosh, S.K., \& Chaudhuri, K.S. (2011). An EOQ model for a deteriorating item with timedependent quadratic demand under permissible delay in payment. Applied Mathematics and Computation, 218, 1- 9.

Jaggi, C.K., Aggarwal, K.K., \& Goel, S.K. (2007). Retailer's optimal ordering policy under two stage trade credit financing. Advanced Modeling and Optimization, 9(1), 67-80.

Jaggi, C. K., Goel, S. K., \& Mittal, M. (2011). Economic order quantity model for deteriorating items with imperfect quality and permissible delay on payment.International Journal of Industrial Engineering Computations, 2(1), 123-140.

Liao, H.C., Tsai, C.H., \& Su, C.T. (2000). An inventory model with deteriorating items under inflation when a delay in payment is permissible. International Journal of Production Economics, 63, 207 214.

Liao, J.J., \& Huang, K.N. (2010).Deterministic inventory model for deteriorating items with trade credit financing and capacity constraints. Computers and Industrial Engineering, 59, 611-618.

Muhlemann, A.P. and Valtis-Spanopoulos, N.P. (1980). A variable holding cost rate EOQ model. European Journal of Operational Research, 4, 132- 135.

Pal, S., Goswami, A., \& Chaudhuri, K.S. (1993). A deterministic inventory model for deteriorating items with stock-dependent demand rate. International Journal of Production Economics, 32(5), 291-299.

Van der Veen, B. (1967) . Introduction to the theory of operational Research. Philips Technical Library, Springer- Verlag, New York.

Weiss, H.J. (1982). Economic order quantity models with non - linear holding cost. European Journal of Operational Research, 9, 56-60.

Roy, M., Gupta, R.K., \& Dasgupta, T. (2012). A technique for determining the optimum mix of logistics service providers of a make-to-order supply chain by formulating and solving a constrained nonlinear cost optimization problem. Decision Science Letters, 2, 95-108.

Sarkar, B. (2012).An EOQ model with delay in payments and time - varying deterioration rate. Mathematical and Computer modeling, 55(3-4), 367-377.

Sana, S.S. (2010). Optimal selling price and lot size with time varying deterioration and partial backlogging. Applied Mathematics and Computation, 217, 185- 194.

Teng, J.T., Chang, C.T., and Goyal, S.K (2005). Optimal pricing and ordering policy under permissible delay in payments. International Journal of Production Economics, 97, 121-129.

Teng, J.T., Min, J., \& Pan, Q. (2012).Economic order quantity model with trade credit financing for non - decreasing demand. Omega, 40, 328-335.

Tripathi, R.P., Misra, S.S, and Shukla, H.S. (2010). A cash flow EOQ model under permissible delay in payments. International Journal of Engineering, Science and Technology, 2(11), 75-84. 